\title{
NICU Protocols of JIPMER: B. Vishnu Bhat, Nishad Plakkal (eds)
}

\section{Published by The Indian Journal of Pediatrics, New Delhi; First Edition; 2020; ISBN: 978-81-938960-7-5; Pages: 465; Price: ₹ 900/-}

\author{
Sridhar Santhanam ${ }^{1}$
}

Received: 24 February 2020 / Accepted: 24 February 2020 /Published online: 16 June 2020

(C) Dr. K C Chaudhuri Foundation 2020

The field of neonatology in India has made great strides over the past two decades. There also is a greater recognition from policy makers on the vital need to improve neonatal health and reduce neonatal/perinatal mortality in the country. This has resulted in a profusion of units caring for newborn in the public and private sector manned by pediatricians, neonatologists and general practitioners. The ultimate goal is to give standardized care with the available resources to assure intact survival of the newborn.

When confronted with a sick newborn with an unexpected issue, the treating physician has to know the best possible management to ensure survival. The NICU Protocols of JIPMER edited by Dr. B. Vishnu Bhat and Dr. Nishad Plakkal will serve as a very good guide to tackle most of the issues facing a doctor manning an SNCU or neonatal ICU. The entire team from JIPMER deserve commendation for this.

The book covers most of the common and uncommon morbidities of the newborn. Topics from resuscitation of newborn to normal newborn care (including Kangaroo mother care) and system wise morbidities are included. All the topics are systematically dealt with including background information, antenatal findings (where appropriate), detailing of the problem and elaboration on the management. Tables, charts, algorithms and photos adequately supplement the information where necessary. The annexure includes a very concise list of common drugs used in neonatology which will prove very useful to the busy practitioner.

Most topics have been extensively dealt with and well referenced. The section on respiratory disorders is elaborate and very practical. Special mention is due for the inclusion of house keeping protocol which is commendable and will be invaluable to doctors running their own neonatal units. The section on sepsis prevention is also not frequently emphasized in protocols and is noteworthy. The TPN calculator shown should also induce all doctors to develop a similar simple calculator to help optimize fluid management of newborn.

No neonatal protocol can be a "one-size-fits-all" guideline for all the units in the country. But if a young practitioner uses this protocol as the basis of his management and modifies according to his environment, he will be on the right track. I am certain that this will help in the daily management of newborns especially in the SNCU's. This book will be a good addition to any unit library.

Publisher's Note Springer Nature remains neutral with regard to jurisdictional claims in published maps and institutional affiliations.

Sridhar Santhanam

santhanamsridhar@gmail.com

1 Department of Neonatology, Christian Medical College, Vellore, Tamil Nadu 632004, India 